\title{
The Plasmid-Borne tet(A) Gene Is an Important Factor Causing Tigecycline Resistance in ST11 Carbapenem-Resistant Klebsiella pneumoniae Under Selective Pressure
}

\author{
Juan $\mathrm{Xu}^{1}$, Zhongliang Zhu' ${ }^{2}$, Yanmin $\mathrm{Chen}^{2}$, Weizhong Wang ${ }^{2}$ and Fang $\mathrm{He}^{2 *}$ \\ 'School of Public Health, Hangzhou Medical College, Hangzhou, China, ${ }^{2}$ Department of Clinical Laboratory, Zhejiang \\ Provincial People's Hospital, People's Hospital of Hangzhou Medical College, Hangzhou, China
}

\section{OPEN ACCESS}

Edited by:

Zhi Ruan,

Zhejiang University, China

Reviewed by:

Ning Dong,

City University of Hong Kong,

Hong Kong

Shangshang Qin,

Zhengzhou University, China

*Correspondence:

Fang $\mathrm{He}$

hetrue@163.com

Specialty section: This article was submitted to Antimicrobials, Resistance and Chemotherapy,

a section of the journal

Frontiers in Microbiology

Received: 22 December 2020 Accepted: 02 February 2021 Published: 24 February 2021

Citation:

$X u$ J, Zhu Z, Chen Y, Wang W and He F (2021) The Plasmid-Borne tet(A) Gene Is an Important Factor Causing

Tigecycline Resistance in ST11

Carbapenem-Resistant Klebsiella pneumoniae Under Selective

Pressure.

Front. Microbiol. 12:644949. doi: 10.3389/fmicb.2021.644949
The emergence and prevalence of tigecycline-resistant Klebsiella pneumoniae have seriously compromised the effectiveness of antimicrobial agents in the treatment of infections. To explore the role of the plasmid-borne tet $(A)$ gene in tigecycline resistance in carbapenem-resistant $K$. pneumoniae (CRKP), a total of $63 \mathrm{CRKP}$ isolates were collected from a tertiary hospital in Hangzhou, China. The minimum inhibitory concentration (MIC) of tigecycline, mutation rate of tet(A) gene, genetic surroundings of tet(A)-carrying transmissible plasmid and the contribution of tet(A) mutation to tigecycline resistance were analyzed using antimicrobial susceptibility test, whole-genome sequencing, tigecycline resistance evolution experiment, and plasmid conjugation experiment. Our results showed that $52.4 \%$ (33 isolates) of the test isolates carried the tet(A) gene; among them, 75.8\% (25 isolates) exhibited a tigecycline nonsusceptible phenotype (MIC $=4 \mathrm{mg} / \mathrm{L}$ ). Three clonal groups (cluster I, cluster II, and cluster III) were identified in these tet(A)-bearing isolates. All 17 isolates belonged to serotype KL21 (cluster I), which differed by only 13 SNPs, suggesting a clonal spread of tet(A)-positive ST11 K. pneumoniae with serotype KL21 occurred in the sampling hospital. The induction of tigecycline resistance experiments showed that $71.4 \%$ of strains evolved tet(A) mutations and developed a high-level tigecycline resistance. Eight amino acid substitutions were identified in these mutants. The most common amino acid substitution was A370V, followed by S251A and G300E. Twelve isolates carrying tet(A) mutants succeeded in the filter mating experiment with a conjugation efficiency of $10^{-3}-10^{-8}$. Tigecycline MICs in E. coli EC600 transconjugants with a mutated tet(A) were 2 to 8-fold higher than those in E. coli EC600 transconjugants with a wild-type tet(A). One ColRNAI/IncFIl type and two IncFIl type tet(A)-bearing conjugative plasmids were identified in this study, including a class 1 integron containing multiple antibiotic resistance genes, i.e., tet(A), gnrS1, bla $\angle A P-2$, catA2, sul2, and dfrA14. Our study revealed the wide-spread situation of plasmid-borne tet(A) gene in clinical $\mathrm{CRKP}$, and mutation of tet $(A)$ is a potential driven force that lead to tigecycline resistance.

Keywords: mutation, plasmid-bearing, tigecycline, tet(A), Klebsiella pneumoniae 


\section{INTRODUCTION}

Carbapenem-resistant Klebsiella pneumoniae (CRKP) is currently a substantial threat to public health worldwide. CRKP can cause a variety of infections, such as pneumonia, liver abscess, urinary tract infection, and bloodstream infection. CRKP often carry multiple antimicrobial resistance genes in the chromosome and plasmids, enabling the strain to be resistant to almost all antibiotics, except colistin and tigecycline. Tigecycline, the first glycylcycline drug, is an extended-spectrum antibiotic that inhibits protein synthesis by binding to the $30 \mathrm{~S}$ ribosome and can overcome the mechanisms of tetracycline resistance (Pankey, 2005).

Antibiotics that can treat CRKP infections are limited. Tigecycline remains an important treatment method for CRKP. However, tigecycline resistance has emerged since the approval of this antibiotic and has been reported frequently in Enterobacteriaceae (Hoban et al., 2005). Previous reports have shown that overexpression of resistance-nodulation-cell division (RND)-type efflux pumps is associated with tigecycline resistance in Enterobacteriaceae, such as AcrAB (Ruzin et al., 2005; Keeney et al., 2007; Bratu et al., 2009; He et al., 2015). Ribosomal protein mutation (via the rpsJ gene) has also been reported to cause tigecycline resistance in Enterobacteriaceae (Beabout et al., 2015; He et al., 2018; Xu et al., 2020). Recently, the plasmid-mediated mobile tigecycline resistance gene tet(X4) and its variants has been reported in Enterobacteriaceae (He et al., 2019; Sun et al., 2019). However, this gene has most often been reported in Escherichia coli strains (Zhang et al., 2020), and its role in K. pneumoniae is limited.

In 2018, we reported the first case of tigecycline resistance in CRKP mediated by tet(A) evolution in vivo during tigecycline treatment (Du et al., 2018). Previously, Linkevicius et al. (2016) observed that evolutionary changes in tet(A) can cause tigecycline resistance in E. coli in vitro. Chiu et al. (2017) considered widespread mutated tet(A) gene to be concerning for the possible dissemination of tigecycline resistance in K. pneumoniae. To explore the role of tet(A) in tigecycline resistance in clinical CRKP isolates, tigecycline minimum inhibitory concentration (MIC) distribution, tet(A)-bearing rate, tet(A) mutation rate and transmission ability of clinical CRKP isolates were analyzed through antimicrobial susceptibility tests, whole genome sequencing, bioinformatics analysis, and plasmid conjugation experiments.

\section{MATERIALS AND METHODS}

\section{CRKP Clinical Isolates}

A total of 63 non-repetitive CRKP clinical strains were continuously collected from April 1st to May 30th in 2018 at a tertiary hospital in Hangzhou, China. Strains from different specimens of the same patient or specimens collected from the same patient at different times were considered to be duplicate strains, and only the first strain was selected for subsequent research. Supplementary Figure 1 outlined the detailed specimen collection information. All of the isolates were identified using the VITEK MS system (bioMérieux, Marcyl'Étoile, France). The carbapenem resistance genes, bla $a_{K P C}$ and $b l a_{N D M}$, as well as the tetracycline resistance gene, tet(A), were amplified by PCR and further sent for Sanger sequencing.

\section{Antimicrobial Susceptibility Test}

Antimicrobial susceptibility testing was conducted using standard broth microdilution tests and the VITEK 2 system (bioMérieux) with Gram-negative antimicrobial susceptibility testing cards (AST-GN13) following the guidelines of the Clinical and Laboratory Standards Institute (CLSI). Antimicrobial agents: amoxicillin/clavulanate, ceftriaxone, cefepime, cefoxitin, aztreonam, piperacillin/tazobactam, imipenem, meropenem, amikacin, levofloxacin, sulfamethoxazole/trimethoprim, colistin, and tigecycline were used in the test. Antimicrobial susceptibility was determined using breakpoints approved by the CLSI (2019). For tigecycline MIC detection, standard broth microdilution tests were adopted with fresh $(<12 \mathrm{~h})$ Mueller-Hinton broth (Cationadjusted, Oxoid Ltd., Basingstoke, Hampshire, United Kingdom). E. coli ATCC 25922 was used for quality control. As there are no CLSI breakpoints for tigecycline, the FDA standard was adopted $^{1}$. The interpretation of colistin MIC was follow by the EUCAST guideline (Breakpoints for 2021) . $^{2}$

\section{Quantitative Real-Time PCR}

mRNA expression levels of the efflux pump genes, acrA and $\operatorname{acr} B$, in tigecycline-resistant isolates were examined by quantitative real-time PCR according to our previously published paper ( $\mathrm{He}$ et al., 2015). The relative expression of each target gene was calibrated against the corresponding expression of K. pneumoniae type strain ATCC 13883 (expression = 1), which served as a control with a tigecycline MIC of $0.125 \mathrm{mg} / \mathrm{L}$. Relative expression levels of the two genes were analyzed by the $2^{-\Delta \Delta} C T$ analytical method.

\section{Whole-Genome Sequencing}

Isolates confirmed to possess the tet $(\mathrm{A})$ gene or resistance to tigecycline were sent for whole-genome sequencing using the Illumina NovaSeq 6000 platform (Illumina Inc., San Diego, CA, United States). In brief, genomic DNA was extracted using a QIAamp DNA Mini Kit (Qiagen, Valencia, CA, United States) and sent for sequencing using the paired-end $2 \times 150$-bp protocol. The draft genome sequences were assembled using SPAdes 3.13.0. Three strains with tet(A) mutants (CRKP52R, CRKP66R, and CRKP78R) were further sent for Nanopore sequencing with a long-read MinION sequencer (Nanopore, Oxford, United Kingdom). Both short Illumina reads and long MinION reads were hybrid assembled using Unicycler (v0.4.7). Complete genome sequences were generated and automatically annotated by the NCBI Prokaryotic Genome Annotation Pipeline (PGAP) server.

\footnotetext{
${ }^{1} \mathrm{https} / /$ www.fda.gov/drugs/development-resources/tigecycline-injectionproducts

${ }^{2}$ https://eucast.org/
} 


\section{Genomic and Phylogenetic Relationship Analysis of tet(A)-Positive Isolates}

MLST, acquired antibiotic resistance genes (ARGs) and plasmid replicons were analyzed using the BacWGSTdb 2.0 server (Ruan and Feng, 2016; Feng et al., 2020; Ruan et al., 2020). The phylogenetic relationship between tet(A)carrying isolates was analyzed using the (neighbor joining $(\mathrm{NJ})$ )/unweighted pair group method with arithmetic mean (UPGMA) phylogeny method (MAFFT version 7) based on a core genome single nucleotide polymorphism strategy (Katoh et al., 2019). A phylogenetic tree was constructed using the resulting SNPs with recombination regions removed using the maximum parsimony algorithm (Jia et al., 2019). The KL type of K. pneumoniae was predicted by Kaptive Web (Wick et al., 2018).

\section{Tigecycline Resistance Evolution Experiment in vitro}

Wild-type tet(A)-carrying CRKP clinical isolates were used as parental strains in tigecycline resistance evolution experiments in vitro. Tigecycline-resistant mutants were selected by successive passages in $\mathrm{MH}$ broth containing increasing concentrations of tigecycline. In brief, one single clone of the parental strain was inoculated in $\mathrm{MH}$ broth overnight, and $200 \mu \mathrm{L}$ of overnight cultures was added to $2 \mathrm{~mL}$ of fresh $\mathrm{MH}$ broth containing serial concentrations of tigecycline. The selective concentration began at $0.5 \mathrm{mg} / \mathrm{L}$ and doubled every $24 \mathrm{~h}$. The protocol was repeated until the mutants grew at a tigecycline concentration of $32 \mathrm{mg} / \mathrm{L}$.

\section{Conjugation Experiment and VITEK MS Identification}

Tigecycline-resistant tet(A) mutants obtained from tigecycline resistance evolution experiments and their parental strains with wild-type tet(A) were used as donors, and rifampicin-resistant E. coli EC600 was used as the recipient. Transconjugants were selected on $\mathrm{MH}$ agar plates supplemented with tetracycline $(16 \mathrm{mg} / \mathrm{L})$ and rifampicin $(600 \mathrm{mg} / \mathrm{L})$. E. coli EC600 transconjugants were identified using the VITEK MS system, and tet $(\mathrm{A})$ gene mutations were further confirmed by PCR and Sanger sequencing. The conjugation efficiency was measured and calculated following the protocol in https://openwetware.org/wiki/conjugation.

\section{Characterization of the tet(A)-Bearing Plasmid and Genetic Background of tet(A)}

Circular comparisons of the tet(A)-carrying plasmid were conducted with BLAST Ring Image Generator (BRIG) based on concentric rings (Alikhan et al., 2011). Insertion elements (ISs) located on the plasmids were predicted by application of ISfinder (Siguier et al., 2006). Integrative and conjugative elements (ICEs) were predicted using ICEberg (Liu et al., 2019). The genetic location and background of tet(A) were determined by aligning the contigs carrying tet(A) with complete genome sequences generated in this study using CLC Genomics Workbench 10.0.1.

\section{Nucleotide Sequence Accession Numbers}

We deposited the complete sequences of the CRKP52R, CRKP66R, and CRKP78R K. pneumoniae strains and plasmids in GenBank under accession numbers CP066249-CP066253, CP063833-CP063838, and CP066254-CP066259. The draft genome sequences of 33 tet $(\mathrm{A})$-positive strains were deposited in GenBank under accession numbers JAEQKY000000000JAEQME000000000. The sequence with GenBank accession number AJ517790 was used as the reference for the wild-type tet(A) gene with the primary start codon of GTG.

\section{RESULTS}

\section{Tigecycline MIC Distribution and Mutations in rpsJ, ramR, and tet(A)}

The antimicrobial susceptibility testing results are presented in Supplementary Table 1. All isolates were multidrug resistant bacteria with a resistance rate greater than $85 \%$ compared to $\beta$ lactams, quinolones and aminoglycosides but were still highly sensitive to colistin. The tigecycline MIC distribution of the 63 isolates is presented in Figure 1A. All 63 isolates carried bla $a_{K P C-2}$ gene, and the in silico MLST analysis showed that all strains, except three ST437 strains, one ST751 strain, and one ST15 strain, belonged to ST11. The highest MIC was $16 \mathrm{mg} / \mathrm{L}$ for isolate CRKP65, and further WGS results showed that this isolate did not harbor the tet(A) gene but had a rpsJ mutation (V57L). One isolate, namely, CRKP26, had a tigecycline MIC of $8 \mathrm{mg} / \mathrm{L}$. Further WGS results showed that this strain had a ramR mutation (GATCCTG insertion at 222-223 resulted in frameshift mutation) and high expression of the RND efflux pump, AcrAB (Table 1), but did not harbor the tet (A) gene. Twenty-six isolates had a MIC of $4 \mathrm{mg} / \mathrm{L}$, and among these, 25 isolates harbored wild-type tet(A). According to the FDA standard (MIC $>2 \mathrm{mg} / \mathrm{L}$ for tigecycline non-susceptible), there were 28 tigecycline nonsusceptible isolates, and the non-susceptible rate was $44.4 \%$. The expression levels of the RND efflux pump genes, acrA and $a c r B$, as well as the mutations in $r p s J, \operatorname{ramR}$, and $\operatorname{tet}(\mathrm{A})$ in tigecycline non-susceptible isolates are presented in Table 1. Four isolates (CRKP5, CRKP15, CRKP21, and CRKP26) had a high expression (two-fold increase compare to reference strain) of the AcrAB efflux pump.

\section{Characterizations of tet(A)-Positive Isolates and Phylogenetic Analysis}

The PCR and Sanger sequencing results showed that 33 of the 63 isolates $(52.4 \%)$ carried the tet $(\mathrm{A})$ gene, all of which were wildtype. The tigecycline MIC distribution of the $33 \operatorname{tet}(\mathrm{A})$-positive isolates is presented in Figure 1B, and there were 25 isolates with MIC $4 \mathrm{mg} / \mathrm{L}$ (75.8\%) and eight isolates (24.2\%) with MIC $2 \mathrm{mg} / \mathrm{L}$. Compared with tet(A)-negative isolates, the tigecycline MIC is generally increased by two-fold. All 33 isolates belonged to ST11. The antimicrobial resistance genes and serotype based on WGS data of these 33 tet(A)-bearing isolates are presented in Figure 2. In total, 16 antimicrobial resistance genes were found in these 


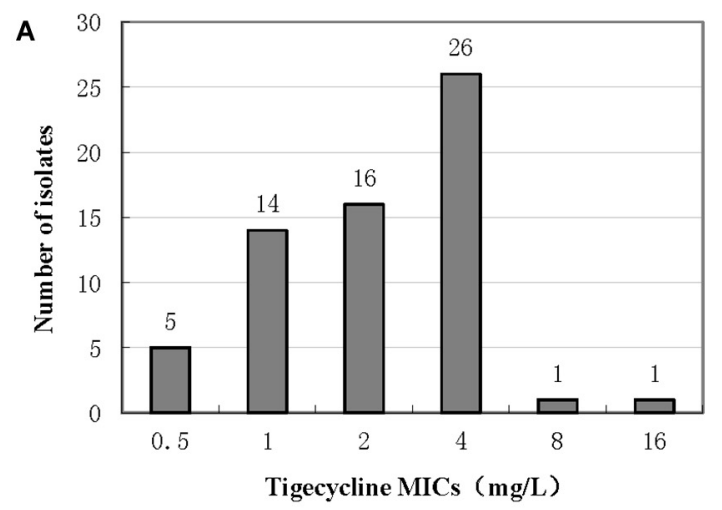

Tigecycline MIC distribution for total 63 CRKP clinical isolates.

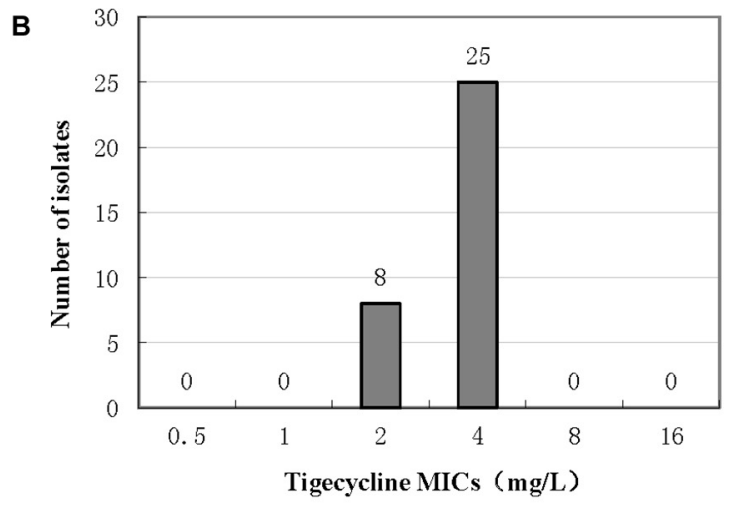

Tigecycline MIC distribution for 33 tetA-bearing isolates.

FIGURE 1 | Tigecycline MIC distributions of $63 \mathrm{CRKP}$ clinical isolates and 33 tet(A)-bearing isolates.

TABLE 1 | Expression levels of the acrA and acrB efflux pump genes and mutation of rpsJ, ramR, and tet(A) in tigecycline non-susceptible isolates.

\begin{tabular}{|c|c|c|c|c|c|c|}
\hline \multirow[t]{2}{*}{ Isolate } & \multirow[t]{2}{*}{$\operatorname{MIC}(m g / L)^{b}$} & \multicolumn{2}{|c|}{ Relative expression ${ }^{a}$} & \multirow[t]{2}{*}{$\operatorname{Tet}(\mathrm{A})^{c}$} & \multicolumn{2}{|c|}{ Mutation } \\
\hline & & acrA & $a c r B$ & & rpsJ & $\operatorname{ram} R$ \\
\hline ATCC 13883 & 0.125 & 1 & 1 & ND & - & - \\
\hline CRKP5 & 4 & $2.16 \pm 0.10$ & $1.66 \pm 0.31$ & WT & - & - \\
\hline CRKP8 & 4 & $1.18 \pm 0.15$ & $1.27 \pm 0.03$ & WT & - & - \\
\hline CRKP10 & 4 & $1.28 \pm 0.11$ & $1.08 \pm 0.23$ & WT & - & - \\
\hline CRKP15 & 4 & $2.38 \pm 0.39$ & $2.46 \pm 0.40$ & WT & - & - \\
\hline CRKP17 & 4 & $1.44 \pm 0.28$ & $0.99 \pm 0.12$ & WT & - & - \\
\hline CRKP21 & 4 & $2.18 \pm 0.15$ & $2.07 \pm 0.45$ & WT & - & - \\
\hline CRKP22 & 4 & $1.03 \pm 0.05$ & $0.81 \pm 0.17$ & WT & - & - \\
\hline CRKP24 & 4 & $0.89 \pm 0.15$ & $0.75 \pm 0.05$ & WT & - & - \\
\hline CRKP26 & 8 & $3.78 \pm 0.29$ & $3.56 \pm 0.24$ & ND & - & GATCCTG insertion at 222-223 \\
\hline CRKP29 & 4 & $1.99 \pm 0.27$ & $1.83 \pm 0.12$ & WT & - & - \\
\hline CRKP31 & 4 & $0.89 \pm 0.20$ & $0.80 \pm 0.16$ & ND & & - \\
\hline CRKP34 & 4 & $1.94 \pm 0.06$ & $1.81 \pm 0.24$ & WT & - & - \\
\hline CRKP38 & 4 & $1.67 \pm 0.30$ & $1.00 \pm 0.22$ & WT & - & - \\
\hline CRKP39 & 4 & $1.42 \pm 0.23$ & $0.71 \pm 0.06$ & WT & - & - \\
\hline CRKP41 & 4 & $1.31 \pm 0.34$ & $0.78 \pm 0.06$ & WT & - & - \\
\hline CRKP42 & 4 & $0.87 \pm 0.12$ & $0.89 \pm 0.12$ & WT & - & - \\
\hline CRKP43 & 4 & $0.78 \pm 0.06$ & $0.67 \pm 0.02$ & WT & - & - \\
\hline CRKP45 & 4 & $1.20 \pm 0.09$ & $0.98 \pm 0.12$ & WT & - & - \\
\hline CRKP51 & 4 & $1.41 \pm 0.28$ & $1.14 \pm 0.10$ & WT & - & - \\
\hline CRKP52 & 4 & $1.35 \pm 0.15$ & $1.13 \pm 0.14$ & WT & - & - \\
\hline CRKP55 & 4 & $1.79 \pm 0.28$ & $1.25 \pm 0.15$ & WT & - & - \\
\hline CRKP59 & 4 & $1.59 \pm 0.60$ & $1.17 \pm 0.19$ & WT & - & - \\
\hline CRKP61 & 4 & $0.81 \pm 0.14$ & $0.77 \pm 0.15$ & WT & - & - \\
\hline CRKP62 & 4 & $1.02 \pm 0.13$ & $0.73 \pm 0.06$ & WT & - & - \\
\hline CRKP65 & 16 & $1.30 \pm 0.25$ & $1.14 \pm 0.03$ & ND & G169C (V57L) & - \\
\hline CRKP72 & 4 & $1.03 \pm 0.15$ & $1.28 \pm 0.28$ & WT & - & - \\
\hline CRKP77 & 4 & $1.91 \pm 0.54$ & $1.32 \pm 0.33$ & WT & - & - \\
\hline CRKP80 & 4 & $1.17 \pm 0.34$ & $0.92 \pm 0.12$ & WT & - & - \\
\hline
\end{tabular}

${ }^{a}$ Relative expression compared with K. pneumoniae type strain ATCC 13883 (expression $=1$ ). Results represent the means of three runs \pm standard deviation.

bigecycline MIC.

${ }^{c} N D$, tet(A) not detected. WT, wild-type tet(A). 

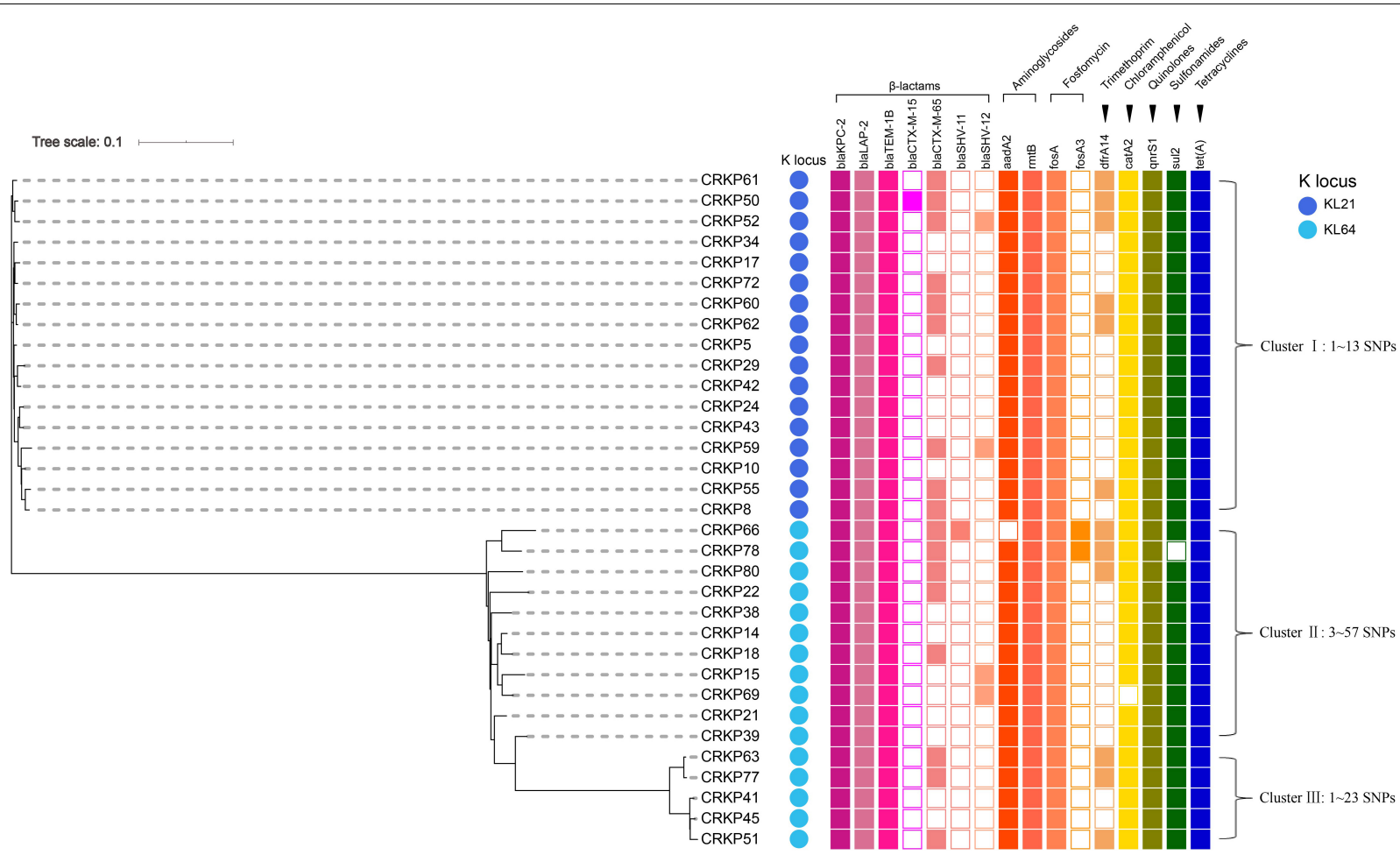

FIGURE 2 | Phylogenetic relationship, antimicrobial resistance genes and serotype analysis based on WGS data of 33 tet(A)-bearing CRKP isolates. The cells in different colors indicate the presence of the antimicrobial resistance gene, while the blank cells indicate the absence of the gene. The color of each circle indicates a distinct capsular serotype.

isolates, including $b l a_{K P C-2}, b l a_{L A P-2}, b l a_{T E M-1 B}$, fos $A, q n r S 1$, $r m t B$, tet $(\mathrm{A}), b l a_{C T X}-M-15, b l a_{C T X}-M-65, b l a_{S H V}-11, b l a_{S H V}-12$, aadA2, dfrA14, catA2, fosA3, and sul2. These isolates were divided into two serotypes (KL21 and KL64). The phylogenetic tree is presented in Figure 2, and SNP differences are presented in Supplementary Figure 2. Three clonal clusters (cluster I, cluster II, and cluster III) were identified. All 17 isolates belonged to serotype KL21 (cluster I), which differed with 13 SNPs. According to the relatedness criteria recommended for SNP typing schemes of K. pneumoniae reported by Schürch et al. (2018), a difference of SNPs $\leq 18$ represents epidemiologically related. Therefore, a clonal spread of tet(A)-positive ST11 K. pneumoniae with serotype KL21 occurred in the sampling hospital.

\section{Mutations of tet(A) in the Tigecycline Resistance Evolution Experiment}

All 33 wild-type tet(A)-carrying CRKP isolates were subjected to tigecycline resistance evolution experiments in vitro. Under successive passages of tigecycline induction in $\mathrm{MH}$ broth for approximately 2 weeks, 28 isolates were induced to develop resistance with a tigecycline MIC $\geq 32 \mathrm{mg} / \mathrm{L}$. PCR and Sanger sequencing detected 20 of the 28 isolates that evolved tet(A) mutations (Table 2), and the mutation rate was $71.4 \%$. The following eight amino acid substitutions were identified in these mutants: A264V, I248L, A370V, S251A, G300E, G300V, A53G, and G237V. The most common amino acid substitution was
A370V, which appeared six times, followed by S251A and G300E (appeared four times each). All 20 tet(A) mutants were subjected to conjugation experiments in which they were used as donors, and E. coli EC600 was the recipient. Twelve mutants succeeded in the conjugation experiment, and the conjugation efficiency was at a frequency of $10^{-3}-10^{-8}$ (Table 2). The MICs of tigecycline and tetracycline of E. coli EC600 transconjugants of tet(A) mutants and wild-type tet(A) are presented in Table 2. In general, tigecycline MICs in E. coli EC600 transconjugants of mutated tet(A) were 2- to 8-fold higher than E. coli EC600 transconjugants of wild-type tet(A), and the MICs of tetracycline also increased in eight strains.

\section{Characterization of the tet(A)-Bearing Plasmid and Genetic Background of tet(A)}

The complete genome sequences of three tet(A) mutants (CRKP52R, CRKP66R, and CRKP78R) were obtained using Nanopore sequencing. CRKP52R, CRKP66R, and CRKP78R were tigecycline-resistant tet(A) mutants collected in the tigecycline resistance evolution experiment. The parental strains of CRKP52R, CRKP66R, and CRKP78R were K. pneumoniae strains CRKP52, CRKP66, and CRKP78, respectively. tet(A) was located on plasmids in these strains, and three tet(A)-bearing plasmids were identified in CRKP52R, CRKP66R, and CRKP78R. The plasmid from CRKP52R was a ColRNAI/IncFII plasmid 
TABLE 2 | Tigecycline and tetracycline MICs of E. coli EC600 transconjugants of tet(A) mutants or wild-type tet(A).

\begin{tabular}{|c|c|c|c|c|c|c|c|c|c|c|}
\hline \multirow[t]{2}{*}{ Isolates } & \multirow[t]{2}{*}{$\begin{array}{l}\mathrm{TGC}^{\mathrm{C}} \mathrm{MICs} \\
(\mathrm{mg} / \mathrm{L})\end{array}$} & \multirow[t]{2}{*}{$\operatorname{tet}(\mathrm{A})^{\mathrm{a}}$} & \multirow[t]{2}{*}{$\begin{array}{l}\text { TGC MICs after tigecycline } \\
\text { induction (mg/L) }\end{array}$} & \multicolumn{2}{|c|}{$\begin{array}{c}\text { tet(A) mutation after tigecycline } \\
\text { induction }^{\mathrm{b}}\end{array}$} & \multirow[t]{2}{*}{$\begin{array}{c}\text { Conjugation } \\
\text { efficiency }\end{array}$} & \multicolumn{2}{|c|}{$\begin{array}{l}\text { E. coli EC600 transconjugant } \\
\text { of tet(A) mutant }^{\mathrm{c}}\end{array}$} & \multicolumn{2}{|c|}{$\begin{array}{l}\text { E. coli EC600 transconjugant } \\
\text { of wild-type tet(A) }{ }^{\mathrm{C}}\end{array}$} \\
\hline & & & & $\begin{array}{l}\text { Nucleotide } \\
\text { change }\end{array}$ & $\begin{array}{l}\text { Amino acid } \\
\text { change }\end{array}$ & & TGC (mg/L) & $\mathrm{TC}(\mathrm{mg} / \mathrm{L})$ & TGC (mg/L) & $\mathrm{TC}(\mathrm{mg} / \mathrm{L})$ \\
\hline CRKP5 & 4 & WT & 64 & - & - & - & - & - & - & - \\
\hline CRKP8 & 4 & WT & 64 & C791T & A264V & $(1.4 \pm 0.6) \times 10^{-4}$ & 1 & 64 & 0.25 & 64 \\
\hline CRKP10 & 4 & WT & 64 & - & - & - & - & - & - & - \\
\hline CRKP14 & 2 & WT & 64 & A742C & I248L & $(4.8 \pm 1.9) \times 10^{-6}$ & 2 & $>256$ & 0.5 & 128 \\
\hline CRKP15 & 4 & WT & 64 & - & - & - & - & - & - & - \\
\hline CRKP17 & 4 & WT & 64 & - & - & - & - & - & - & - \\
\hline CRKP18 & 2 & WT & 64 & - & - & - & - & - & - & - \\
\hline CRKP21 & 4 & WT & 64 & - & - & - & - & - & - & - \\
\hline CRKP22 & 4 & WT & 4 & - & - & - & - & - & - & - \\
\hline CRKP24 & 4 & WT & 64 & C1109T & A370V & Failed & NA & NA & NA & NA \\
\hline CRKP29 & 4 & WT & 64 & $\mathrm{~T} 751 \mathrm{G}$ & S251A & $(6.0 \pm 3.4) \times 10^{-6}$ & 1 & 256 & 0.25 & 128 \\
\hline CRKP34 & 4 & WT & 4 & - & - & - & - & - & - & - \\
\hline CRKP38 & 4 & WT & 64 & $\mathrm{~T} 751 \mathrm{G}$ & S251A & $(1.8 \pm 0.9) \times 10^{-3}$ & 1 & 64 & 0.25 & 64 \\
\hline CRKP39 & 4 & WT & 64 & G899T & G300V & Failed & NA & NA & NA & NA \\
\hline CRKP41 & 4 & WT & 4 & - & - & - & - & - & - & - \\
\hline CRKP42 & 4 & WT & 64 & C1109T & A370V & Failed & NA & NA & NA & NA \\
\hline CRKP43 & 4 & WT & 64 & G710T T751G & G237V S251A & Failed & NA & NA & NA & NA \\
\hline CRKP45 & 4 & WT & 4 & - & - & - & - & - & - & - \\
\hline CRKP50 & 2 & WT & 64 & C791T & A264V & $(7.7 \pm 3.3) \times 10^{-7}$ & 1 & 128 & 0.5 & 64 \\
\hline CRKP51 & 4 & WT & 64 & G899A & G300E & $(3.7 \pm 1.9) \times 10^{-6}$ & 2 & $>256$ & 0.25 & 128 \\
\hline CRKP52 & 4 & WT & 64 & G899A & G300E & $(5.2 \pm 2.5) \times 10^{-8}$ & 1 & 256 & 0.25 & 128 \\
\hline CRKP55 & 4 & WT & 64 & C1109T & A370V & $(3.2 \pm 1.4) \times 10^{-5}$ & 1 & 128 & 0.5 & 128 \\
\hline CRKP59 & 4 & WT & 64 & C1109T & A370V & $(1.1 \pm 0.7) \times 10^{-5}$ & 1 & $>256$ & 0.25 & 128 \\
\hline CRKP60 & 2 & WT & 64 & G899A & G300E & Failed & NA & NA & NA & NA \\
\hline CRKP61 & 4 & WT & 64 & - & - & - & - & - & - & - \\
\hline CRKP62 & 4 & WT & 4 & - & - & - & - & - & - & - \\
\hline CRKP63 & 2 & WT & 32 & - & - & - & - & - & - & - \\
\hline CRKP66 & 2 & WT & 64 & С1109т & A370V & $(1.4 \pm 1.2) \times 10^{-6}$ & 1 & 64 & 0.25 & 64 \\
\hline CRKP69 & 2 & WT & 64 & G899A & G300E & $(1.1 \pm 0.5) \times 10^{-4}$ & 4 & $>256$ & 0.5 & 128 \\
\hline CRKP72 & 4 & WT & 64 & C158G & A53G & Failed & NA & NA & NA & NA \\
\hline CRKP77 & 4 & WT & 64 & C1109T & A370V & Failed & NA & NA & NA & NA \\
\hline CRKP78 & 2 & WT & 64 & $\mathrm{~T} 751 \mathrm{G}$ & S251A & $(4.7 \pm 2.8) \times 10^{-6}$ & 4 & $>256$ & 0.5 & 128 \\
\hline CRKP80 & 4 & WT & 64 & C791T & A264V & Failed & NA & NA & NA & NA \\
\hline
\end{tabular}

${ }^{a} W T$, wild-type tet(A).

$b \rightarrow$, no mutation detected in tet(A).

${ }^{C}$ TGC, Tigecycline; TC, Tetracycline. NA, Data not available. 
that was 99,066 bp in size and was designated pCRKP52R4-tetA. Plasmids from CRKP66R and CRKP78R belonged to the IncFII type with sizes of 87,095 and 86,962 bp, and they were designated pCRKP66R-4-tetA and pCRKP78R-4-tetA, respectively (Table 3 ). Similar tet(A)-bearing plasmids in the NCBI GenBank database were searched with the Basic Local Alignment Search Tool (BLAST). We found that IncFII-type and IncFIB-type plasmids with sizes of approximately 90 and
$120 \mathrm{~kb}$ were common plasmids carrying tet(A) in K. pneumoniae (Table 3). The similarity of the plasmid backbone of these plasmids is presented in Figure 3. These IncFII-type plasmids have a plasmid backbone similar to that of ARGs, including $q n r S 1, b l a_{L A P-2}, \operatorname{tet}(\mathrm{A}), \operatorname{cat} A 2, \operatorname{sul} 2$, and $d f r A 14$. The tet(A) genes in the pCRKP52R-4-tetA plasmid were flanked by the qnrS1 and bla $a_{L A P-2}$ resistance genes on the left and the catA2, sul2, and $d f r A 14$ resistance genes on the right, and

TABLE 3 | Detailed information of tet(A)-bearing plasmids obtained in this study and the NCBI database.

\begin{tabular}{|c|c|c|c|c|c|}
\hline Plasmid name & Plasmid replicon & Plasmid size & Host bacteria & Antimicrobial resistance genes & Accession number \\
\hline pCRKP52R-4-tetA & ColRNAI/IncFII & 99066bp & K. pneumoniae & anrS1, bla $L A P-2$, tet(A), catA2, sul2, dfrA14 & CP066252 (this study) \\
\hline pCRKP66R-4-tetA & IncFll & 87095bp & K. pneumoniae & qnrS1, bla ${ }_{L A P-2}$, tet(A), catA2, sul2, dfrA14 & CP063836 (this study) \\
\hline pCRKP78R-4-tetA & IncFl| & 86962bp & K. pneumoniae & qnrS1, bla $L A P-2$, tet(A), catA2, sul2, dfrA14 & CP066257 (this study) \\
\hline pKP18-3-8-IncFII & IncFll & 87095bp & K. pneumoniae & qnrS1, bla $L A P-2$, tet(A), catA2, sul2, dfrA14 & MT035876 (NCBI) \\
\hline pKP18-2079_tetA & IncFll & 84699bp & K. pneumoniae & qnrS1, bla $2 A P-2$, tet(A), sul2, dfrA14 & MT090960 (NCBI) \\
\hline pKP13-53-tet(A) & $\mathrm{Col} / \mathrm{IncFIB}$ & $181383 b p$ & K. pneumoniae & anrS1, bla $2 A P-2, \operatorname{tet}(\mathrm{A})$, sul1, dfrA1, aac(3)-Ild & MN268580 (NCBI) \\
\hline p71221-tetA & IncFIB & $128170 b p$ & K. pneumoniae & tet(A), sul1, dfrA1, mph(A), blasHV-12, aph(3')-la & MN310374 (NCBI) \\
\hline pW08291-tetA & IncFIB & $130483 b p$ & K. pneumoniae & tet(A), sul1, dfrA1, mph(A), blasHV-12 & MN310376 (NCBI) \\
\hline
\end{tabular}

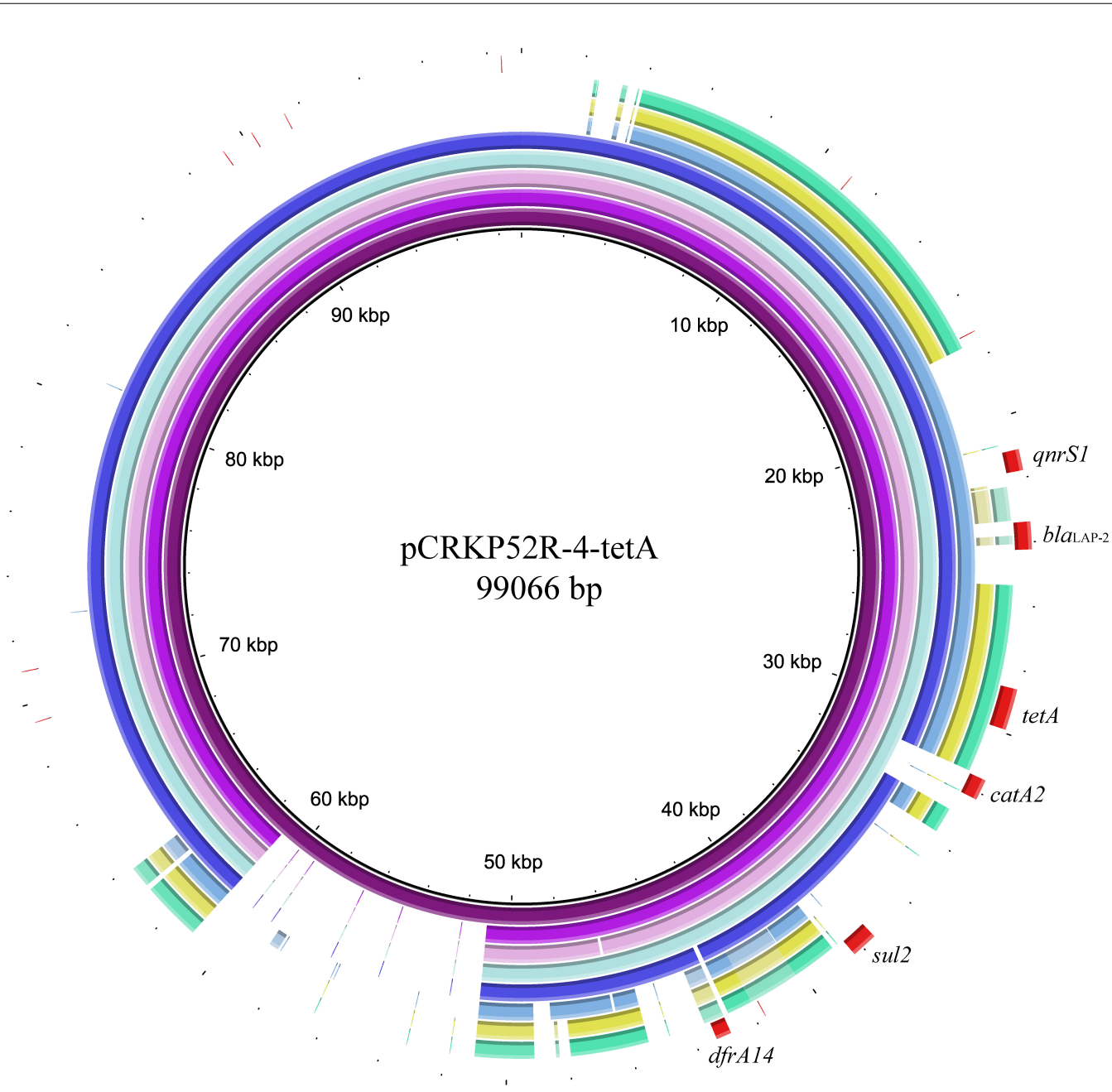

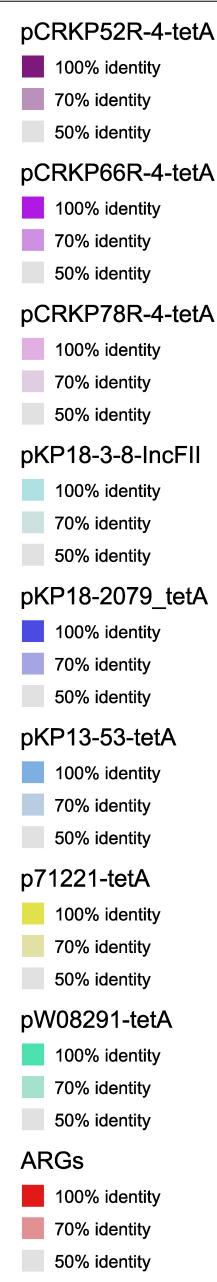

FIGURE 3 | Plasmid backbone comparisons of tet(A)-carrying plasmids. Plasmid information is presented in Table $\mathbf{3}$. ARGs are indicated in red. 
they were all located in ICEs ranging from 11 to $99 \mathrm{~kb}$ in size (Figure 3). One class 1 integron (IntI1) was identified in pCRKP52R-4-tetA (from 18944 to 46168 bp), pCRKP66R-4tetA, and pCRKP78R-4-tetA (Figure 4). This integron contains multiple ARGs, including tet(A). The tet(A) gene was located in the genetic environment, IS26-tetR-tet(A)-eamA-orf-TnAs1, suggesting that it was acquired by horizontal gene transfer with mobilizable transposons.

\section{DISCUSSION}

Carbapenem-resistant $K$. pneumoniae has emerged as an important pathogen worldwide, and the emergence of tigecycline and colistin resistance makes clinical treatment difficult. According to the data obtained in this study, 52.4\% of the tested isolates carried the wild-type tet(A) gene. Among these isolates, $75.8 \%$ of these tet(A)-bearing isolates exhibited a tigecycline nonsusceptible phenotype. No mutations of rps and $\mathrm{ramR}$ genes were identified in these isolates. We also searched the plasmidencoded RND efflux pump genes tmexCD1-toprJ1 in the genome of these strains, but no related genes were found. Compared with tet(A)-negative isolates, the tigecycline MIC was generally increased by approximately two-fold in these wild-type tet(A)bearing isolates. Except for three isolates (CRKP5, CRKP15, and CRKP21) had a high expression of the AcrAB efflux pump (Table 1), we considered that other undiscovered mechanisms may be utilized in these isolates that work together with wildtype tet(A) to mediate tigecycline non-susceptibility. A recent study reported that TetA in synergy with RND-type efflux transporters contribute to tigecycline resistance in Acinetobacter baumannii (Foong et al., 2020). This synergy may also exist in K. pneumoniae, which warrant further investigation.
tet(A) is a MFS family efflux pump, and mutation in tet(A) might result in increased accumulation of tigecycline as a substrate, thus contributing to tigecycline resistance (Linkevicius et al., 2016; Chiu et al., 2017). tet(A)-bearing K. pneumoniae tended to more easily evolve tigecycline resistance under selective pressure as $71.4 \%$ of the strains evolved tet(A) mutations and developed high-level tigecycline resistance in our tigecycline resistance evolution experiment in vitro. We have previously confirmed the contribution of the S251A Tet(A) variant to tigecycline resistance by transformation experiments (Du et al., 2018). Linkevicius et al. (2016) also confirmed that tet(A) mutants of I235F, I248L, S251A, and G300E show increased tigecycline MICs compared to the unmutagenized control. We further conducted transformation experiments on several other mutants (A264V, A370V, G300V, and A53G) identified in this study, and we found these mutants increased the tigecycline MIC in E. coli DH5 $\alpha$ by 2 to 4 -fold compared to the wild-type tet(A) control. The degree of tigecycline MIC increase in E. coli EC600 transconjugants of tet(A) mutants was diverse (Table 2), especially for a few transconjugants harboring the same mutation site (e.g., CRKP29, CRKP38, and CRKP78). Linkevicius et al. (2016) confirmed that the magnitude increase of tigecycline MIC depends on the expression level of the tet(A) mutant. Thus, we speculated that the difference of the tet(A) mutant expression level may be due to the diverse tigecycline MICs in these E. coli EC600 transconjugants. tet(A) mutants are often located in different types of plasmids, and these plasmids have different promoter and regulatory sequences that may result in different expression levels of tet(A).

Multiple types of tet(A)-bearing plasmids were retrieved from the NCBI GenBank database, and circular comparison analysis revealed that they have some similar structures, suggesting

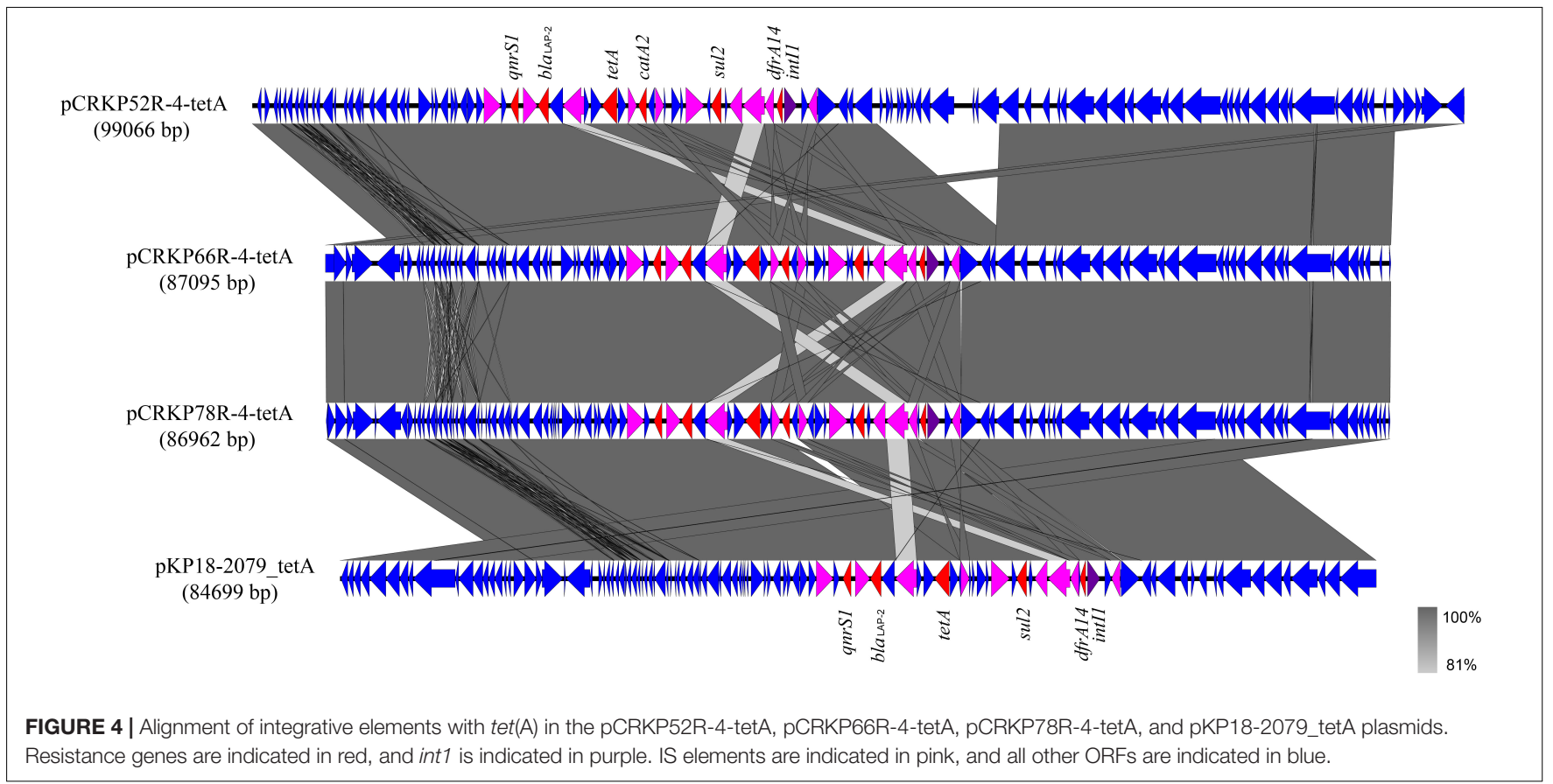


that genetic exchange and recombination among different types of tet(A)-bearing plasmids have occurred (Ribera et al., 2003; Szmolka et al., 2015; Yao et al., 2020). In the three tet(A)bearing plasmids obtained in this study, one class 1 integron containing multiple ARGs, including tet(A), was detected. In addition, tet(A) mutation occurring under selective pressure may lead to tigecycline treatment failure. Zhang et al. (2019) reported the coexistence of $m c r-1$ and the tet(A) variant on the same plasmid from a $K$. pneumoniae isolate in human gut, and Yao et al. (2020) also reported an IncFII plasmid co-harboring bla $a_{I M P-26}$ and tet(A) variant in a clinical $K$. pneumoniae isolate. It seems that tet(A) mutants can not only occur in bla $a_{K P C-2}$-carrying plasmids, but also form fusion plasmids with other carbapenem resistance genes and mor gene, which will cause a higher transmission risk of simultaneous resistance to carbapenem, colistin and tigecycline. The emergence and spread of such fusion plasmid needs our attention.

\section{CONCLUSION}

In conclusion, our study revealed the wide-spread of plasmidborne tet(A) gene in clinical CRKP, and mutation of tet(A) is a potential threat that lead to tigecycline resistance. More attention should be devoted to monitoring the spread of plasmidborne tet(A) in $K$. pneumoniae clinical isolates, especially the emergence of tet(A) mutants. Strict administration of tigecycline and classification management of antibiotics must be carried out with precautions.

\section{DATA AVAILABILITY STATEMENT}

The data presented in the study are deposited in the GenBank repository, accession numbers CP066249-CP066253, CP063833CP063838, CP066254-CP066259, and JAEQKY000000000JAEQME000000000.

\section{REFERENCES}

Alikhan, N. F., Petty, N. K., Ben Zakour, N. L., and Beatson, S. A. (2011). BLAST Ring Image Generator (BRIG): simple prokaryote genome comparisons. BMC Genomics 12:402. doi: 10.1186/1471-2164-12-402

Beabout, K., Hammerstrom, T. G., Perez, A. M., Magalhaes, B. F., Prater, A. G., Clements, T. P., et al. (2015). The ribosomal S10 protein is a general target for decreased tigecycline susceptibility. Antimicrob. Agents Chemother. 59, 5561-5566. doi: 10.1128/aac.00547-15

Bratu, S., Landman, D., George, A., Salvani, J., and Quale, J. (2009). Correlation of the expression of acrB and the regulatory genes marA, soxS and ramA with antimicrobial resistance in clinical isolates of Klebsiella pneumoniae endemic to New York City. J. Antimicrob. Chemother. 64, 278-283. doi: 10.1093/jac/ dkp186

Chiu, S. K., Huang, L. Y., Chen, H., Tsai, Y. K., Liou, C. H., Lin, J. C., et al. (2017). Roles of ramR and tet(A) mutations in conferring tigecycline resistance in carbapenem-resistant Klebsiella pneumoniae clinical isolates. Antimicrob. Agents Chemother. 61:e00391-17.

\section{ETHICS STATEMENT}

This study was conducted in accordance with the Declaration of Helsinki and approved by the Ethics Committee of Zhejiang Provincial People's Hospital. Written informed consent from the patients was exempted by the Ethics Committee of Zhejiang Provincial People's Hospital because the present study only focused on bacteria.

\section{AUTHOR CONTRIBUTIONS}

FH designed the experiments. JX and ZZ performed the experiments and were the major contributors in writing the manuscript. YC and WW analyzed the data. All authors read and approved the final manuscript.

\section{FUNDING}

This study was supported by grants from the National Natural Science Foundation of China (81702042), Natural Science Foundation of Zhejiang Province (LQ19H200003), Natural Science Foundation of Zhejiang Provincial Department of Education (Y202044471), and Zhejiang Provincial Medical and Health Science and Technology Plan (2019KY311).

\section{SUPPLEMENTARY MATERIAL}

The Supplementary Material for this article can be found online at: https://www.frontiersin.org/articles/10.3389/fmicb. 2021.644949/full\#supplementary-material

Supplementary Figure 1 | Specimen type and department distribution of 63 non-repetitive CRKP clinical isolates.

Supplementary Figure 2 | Phylogenetic analysis of 33 tet(A)-bearing isolates based on the core genome SNP strategy.

CLSI (2019). Performance Standards for Antimicrobial Susceptibility Testing: Clinical and Laboratory Standards Institute (CLSI) supplement M100, 29th Edn. Wayne, PA: Clinical and Laboratory Standards Institute.

Du, X., He, F., Shi, Q., Zhao, F., Xu, J., Fu, Y., et al. (2018). The rapid emergence of tigecycline resistance in blaKPC-2 harboring Klebsiella pneumoniae, as mediated in vivo by mutation in tetA during tigecycline treatment. Front. Microbiol. 9:648. doi: 10.3389/fmicb.2018.00648

Feng, Y., Zou, S., Chen, H., Yu, Y., and Ruan, Z. (2020). BacWGSTdb 2.0: a one-stop repository for bacterial whole-genome sequence typing and source tracking. Nucleic Acids Res. 49, D644-D650.

Foong, W. E., Wilhelm, J., Tam, H. K., and Pos, K. M. (2020). Tigecycline efflux in Acinetobacter baumannii is mediated by TetA in synergy with RND-type efflux transporters. J. Antimicrob. Chemother. 75, 1135-1139. doi: 10.1093/jac/ dkaa015

He, F., Fu, Y., Chen, Q., Ruan, Z., Hua, X., Zhou, H., et al. (2015). Tigecycline susceptibility and the role of efflux pumps in tigecycline resistance in KPCproducing Klebsiella pneumoniae. PLoS One 10:e0119064. doi: 10.1371/journal. pone.0119064 
He, F., Shi, Q., Fu, Y., Xu, J., Yu, Y., and Du, X. (2018). Tigecycline resistance caused by rpsJ evolution in a 59-year-old male patient infected with KPCproducing Klebsiella pneumoniae during tigecycline treatment. Infect. Genet. Evol. 66, 188-191. doi: 10.1016/j.meegid.2018.09.025

He, T., Wang, R., Liu, D., Walsh, T. r., Zhang, R., Lv, Y., et al. (2019). Emergence of plasmid-mediated high-level tigecycline resistance genes in animals and humans. Nat. Microbiol. 4, 1450-1456. doi: 10.1038/s41564-019-0445-2

Hoban, D. J., Bouchillon, S. K., Johnson, B. M., Johnson, J. L., and Dowzicky, M. J. (2005). In vitro activity of tigecycline against 6792 gram-negative and grampositive clinical isolates from the global tigecycline evaluation and surveillance trial (TEST Program, 2004). Diagn. Microbiol. Infect. Dis. 52, 215-227. doi: 10.1016/j.diagmicrobio.2005.06.001

Jia, H., Chen, Y., Wang, J., Xie, X., and Ruan, Z. (2019). Emerging challenges of whole-genome-sequencing-powered epidemiological surveillance of globally distributed clonal groups of bacterial infections, giving Acinetobacter baumannii ST195 as an example. Int. J. Med. Microbiol. 309:151339. doi: 10.1016/j.ijmm.2019.151339

Katoh, K., Rozewicki, J., and Yamada, K. D. (2019). MAFFT online service: multiple sequence alignment, interactive sequence choice and visualization. Brief. Bioinform. 20, 1160-1166. doi: 10.1093/bib/bbx108

Keeney, D., Ruzin, A., and Bradford, P. A. (2007). RamA, a transcriptional regulator, and $A c r A B$, an RND-type efflux pump, are associated with decreased susceptibility to tigecycline in Enterobacter cloacae. Microb. Drug Resist. 13, 1-6. doi: 10.1089/mdr.2006.9990

Linkevicius, M., Sandegren, L., and Andersson, D. I. (2016). Potential of tetracycline resistance proteins to evolve tigecycline resistance. Antimicrob. Agents Chemother. 60, 789-796. doi: 10.1128/aac.02465-15

Liu, M., Li, X., Xie, Y., Bi, D., Sun, J., Li, J., et al. (2019). ICEberg 2.0: an updated database of bacterial integrative and conjugative elements. Nucleic Acids Res. 47, D660-D665.

Pankey, G. A. (2005). Tigecycline. J. Antimicrob. Chemother. 56, 470-480.

Ribera, A., Roca, I., Ruiz, J., Gibert, I., and Vila, J. (2003). Partial characterization of a transposon containing the tet(A) determinant in a clinical isolate of Acinetobacter baumannii. J. Antimicrob. Chemother. 52, 477-480. doi: 10.1093/ $\mathrm{jac} / \mathrm{dkg} 344$

Ruan, Z., and Feng, Y. (2016). BacWGSTdb, a database for genotyping and source tracking bacterial pathogens. Nucleic Acids Res. 44, D682-D687.

Ruan, Z., Yu, Y., and Feng, Y. (2020). The global dissemination of bacterial infections necessitates the study of reverse genomic epidemiology. Brief. Bioinform. 21, 741-750. doi: 10.1093/bib/bbz010

Ruzin, A., Visalli, M. A., Keeney, D., and Bradford, P. A. (2005). Influence of transcriptional activator RamA on expression of multidrug efflux pump AcrAB and tigecycline susceptibility in Klebsiella pneumoniae. Antimicrob. Agents Chemother. 49, 1017-1022. doi: 10.1128/aac.49.3.1017-1022.2005
Schürch, A., Arredondo-Alonso, S., Willems, R., and Goering, R. (2018). Whole genome sequencing options for bacterial strain typing and epidemiologic analysis based on single nucleotide polymorphism versus gene-by-gene-based approaches. Clin. Microbiol. Infect. 24, 350-354. doi: 10.1016/j.cmi.2017.12.016

Siguier, P., Perochon, J., Lestrade, L., Mahillon, J., and Chandler, M. (2006). ISfinder: the reference centre for bacterial insertion sequences. Nucleic Acids Res. 34, D32-D36.

Sun, J., Chen, C., Cui, C. y, Zhang, Y., Liu, X., Cui, Z. h, et al. (2019). Plasmid-encoded tet(X) genes that confer high-level tigecycline resistance in Escherichia coli. Nat. Microbiol. 4, 1457-1464. doi: 10.1038/s41564-0190496-4

Szmolka, A., Lestár, B., Pászti, J., Fekete, P., and Nagy, B. (2015). Conjugative IncF and IncI1 plasmids with tet(A) and class 1 integron conferring multidrug resistance in F18(+) porcine enterotoxigenic E. coli. Acta Vet. Hung. 63, 425443. doi: 10.1556/004.2015.040

Wick, R. R., Heinz, E., Holt, K. E., and Wyres, K. L. (2018). Kaptive web: user-friendly capsule and lipopolysaccharide serotype prediction for Klebsiella genomes. J. Clin. Microbiol. 56:e00197-18.

Xu, J., Zhao, Z., Ge, Y., and He, F. (2020). Rapid emergence of a pandrug-resistant Klebsiella pneumoniae ST11 isolate in an inpatient in a teaching hospital in China after treatment with multiple broad-spectrum antibiotics. Infect Drug Resist. 13, 799-804. doi: 10.2147/idr.s243334

Yao, H., Cheng, J., Li, A., Yu, R., Zhao, W., Qin, S., et al. (2020). Molecular characterization of an IncFII(k) plasmid co-harboring bla (IMP-26) and tet(A) variant in a clinical Klebsiella pneumoniae isolate. Front. Microbiol. 11:1610. doi: $10.3389 /$ fmicb.2020.01610

Zhang, R., Dong, N., Shen, Z., Zeng, Y., Lu, J., Liu, C., et al. (2020). Epidemiological and phylogenetic analysis reveals Flavobacteriaceae as potential ancestral source of tigecycline resistance gene tet(X). Nat. Commun. 11:4648.

Zhang, R., Hu, Y. Y., Zhou, H. W., Wang, S. L., Shu, L. B., and Chen, G. X. (2019). Emergence of mcr-1 and the tet(A) variant in a Klebsiella pneumoniae isolate from the faeces of a healthy person. J. Med. Microbiol. 68, 1267-1268. doi: 10.1099/jmm.0.000932

Conflict of Interest: The authors declare that the research was conducted in the absence of any commercial or financial relationships that could be construed as a potential conflict of interest.

Copyright (c) $2021 \mathrm{Xu}$, Zhu, Chen, Wang and He. This is an open-access article distributed under the terms of the Creative Commons Attribution License (CC BY). The use, distribution or reproduction in other forums is permitted, provided the original author(s) and the copyright owner(s) are credited and that the original publication in this journal is cited, in accordance with accepted academic practice. No use, distribution or reproduction is permitted which does not comply with these terms. 